\title{
Preprocessing and Visualization of the VVER Reactor Operational Parameters Archive
}

\author{
S. Ten¹, A.M. Zagrebayev², V.V. Pilyugin³ \\ National Research Nuclear University MEPhI, Kashirskoe hwy 31, 115409, \\ Moscow, Russia \\ 1 ORCID: 00oo-0oo1-9279-1732, tenstanislav@email.com \\ 2 ORCID: oooo-0003-0576-3587, AMZagrebayev@mephi.ru \\ 3 ORCID: 000o-0001-8648-1690, VVPilyugin@mephi.ru
}

\begin{abstract}
For the most part, the solution of the problem of visualizing any data depends on the structure, size and type of data provided. In this work, the data are archives of RBMK or VVER reactors provided from different control and protection systems of the reactors. Despite the fact that several visualization complexes have already been developed, the visualization task is still relevant due to the necessity to improve the quality of monitoring systems and operational personnel.

This paper describes the mathematical apparatus for express analysis of the archive of operational parameters of a VVER nuclear reactor. The developed software makes it possible to carry out express analysis of the VVER reactor archive in terms of plotting altitude or time graphs, as well as using dynamic visualization using the Chernoff faces method, which in turn can provide scientific and practical benefits due to improvement the work quality of operating personnel and conduction analysis of situations that requires additional attention and more detailed analysis.

Also, the fundamental concept of this work is the method of scientific visualization, which is widely used in various theoretical and experimental studies. It can be said that the main aim of scientific visualization is to make invisible visible.

Keywords: Operational Parameters Archive, Scientific Visualization, Data Analysis, Express Analysis, Nuclear Reactor.

\section{Introduction}

Often, when conducting scientific research related to the peculiarities of the operation of a nuclear reactor, one encounters the tasks of analyzing archived data. For example, power plant workers can analyze existing archives in order to adjust the further operation of the reactor. At the same time, during the data analysis, it is possible to identify anomalies in the operation of the reactor and, by conducting a study on the causes of the occurrence of such behavior, prevent their further manifestations, thereby increasing the safety of operation of the reactor itself. Also, when using additional support, operators of nuclear power plants can increase the safety of facilities by quickly responding to changes in parameters or exceeding any of the parameters of the maximum permissible values.

The safe operation of powerful nuclear power reactors is ensured by the availability of information and computational systems that allow measuring, calculating, and monitoring the most important parameters of a nuclear power unit.

At the stations, there is a station-wide data exchange network between the process control systems. Information from various systems enters the data warehouse at regular intervals. Further, the workers of the power unit analyze the data obtained and correct the operation of the reactor.
\end{abstract}


The files located in the data warehouse are not convenient for analysis, therefore, the task arises of forming such a data warehouse into which an archive containing the values of the main parameters of the power unit functioning according to the selected sections would be loaded. The archival information accumulated in this repository can be further used for various studies.

Depending on the task, the requirements for the nature, type and volume of stored information, as well as the degree of its granularity, may differ significantly. For example, to solve the problem of assessing the quality of the work of operating personnel, it is necessary to store information on the type and number of monitored parameters, the values of which have gone beyond the limits established by the regulations, the number and type of operator impacts on the control object (movements of the control and protection system organs (CPS), adjustment of the coolant flow rate, etc.), the degree of spatial stability of the threedimensional energy release field, etc. for several hours. When solving the problem of identifying the causes of failure, for example, fuel elements, fuel assemblies and cassettes, information for a period from several days to several years may be required. In this case, the prehistory of behavior of limiting parameters of the reactor and the power unit may be of interest. For example, for a RBMK-type reactor (high-power channel-type reactor), such parameters as the power of each channel, its power generation, coolant flow through the channel, indications of the systems for monitoring the tightness of the cladding of fuel elements (CFE) and monitoring the integrity of technological channels (TC) [5], linear load on the fuel element, stock up to the crisis of heat exchange, the number of permutations of the fuel cartridge, etc. For a VVER-type reactor (water-water power reactor), the limiting parameters partially coincide with the above, but also contain significant additions and differences associated with the difference in the design of the reactors. For example, the limiting parameter is the concentration of boric acid, the absence of boiling of the coolant, the pressure in the reactor, etc.

However, despite the fact that the tasks listed above are of different nature, the archive of operational parameters of RBMK and VVER reactors should allow solving each of them and, moreover, serve as an information base for solving newly arising problems. With the current level of development of computing systems at NPPs (nuclear power plants), it is possible to organize the storage of all experimental and calculated information with high detail in time over a long period of operation, however, problems arise with the express analysis of a large amount of data.

The fundamental concept in the development of software for the express analysis of an archive is the method of scientific visualization. Scientific visualization is a modern effective approach to data analysis that allows visualizing arrays of data of different nature - abstract or real. Visual information is better perceived and allows to convey the result to the user quickly and efficiently. Physiologically, the perception of visual information is fundamental for humans. The success of visualization directly depends on the correctness of its application, namely on the precise structuring of the approach and the data itself. The essence of the scientific visualization method lies in the fact that the initial analyzed data is associated with some of their static or dynamic graphic interpretation, which is visually analyzed, and the results of the analysis of this graphic interpretation are then interpreted in relation to the original data.

\section{Research of existing software packages}

The paper [9] describes a modern storage of parameters of a nuclear power unit with an RBMK reactor and a specially created module for visualizing archived data in a user-friendly form for viewing the archive database from remote workplaces. This module also serves to easily export data for subsequent analysis and calculations.

The module interface is shown in Fig. 1. At startup, the main window of the program opens, the current state of the database is analyzed and a list of available "time slices" is 
formed. The title of the main window displays information about the time of the last update. There is a button on the toolbar to manually update the list of available slices. In the center of the program window, a cartogram of the parameter that is selected in the list on the left is displayed. Periodically (by default, once every 30 seconds) the program automatically updates the list of available slices.

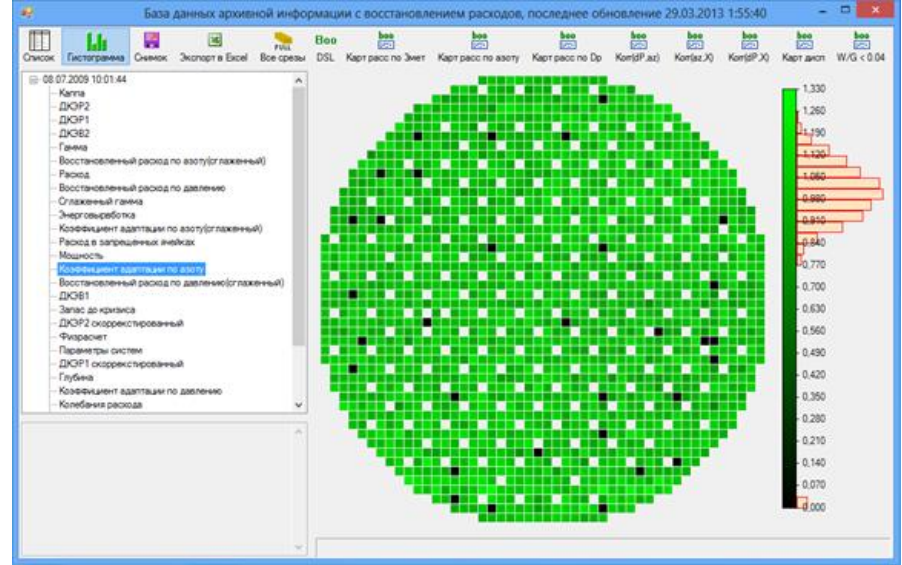

Figure 1: The window of the visualization module after selecting the required information

The data of the SKALA-MICRO system are used to extract such parameters as the reactor loading, the coolant flow rate in each channel, information on the position of the control rods, the readings of the energy release control sensors, as well as the calculated parameters - the reactor power, the power generation of the cassettes and the margin before the crisis.

In many respects, a similar approach was used by us in the development of software for creating an archive of operational parameters of a power unit with a VVER-type reactor. In [10], a software package is described that allows to load archives of VVER reactor parameters into the database and view the already loaded data in a convenient user interface.

The display of data on the $3 \mathrm{D}$ core model has been optimized by simplifying the cell models. To display the parameter values, two separate visual modules have been developed: a table and a 3D model of the VVER reactor core.

On the left in all modules there is a tree-like display of the state of the database (a complete set of loaded data). The data in the tree view is structured as follows: campaignslice-parameter-level (if there are several levels). Below the tree-like display there is a window for displaying extended information on the cell to which the mouse is pointing. Fig. 2 shows the main window of the application.

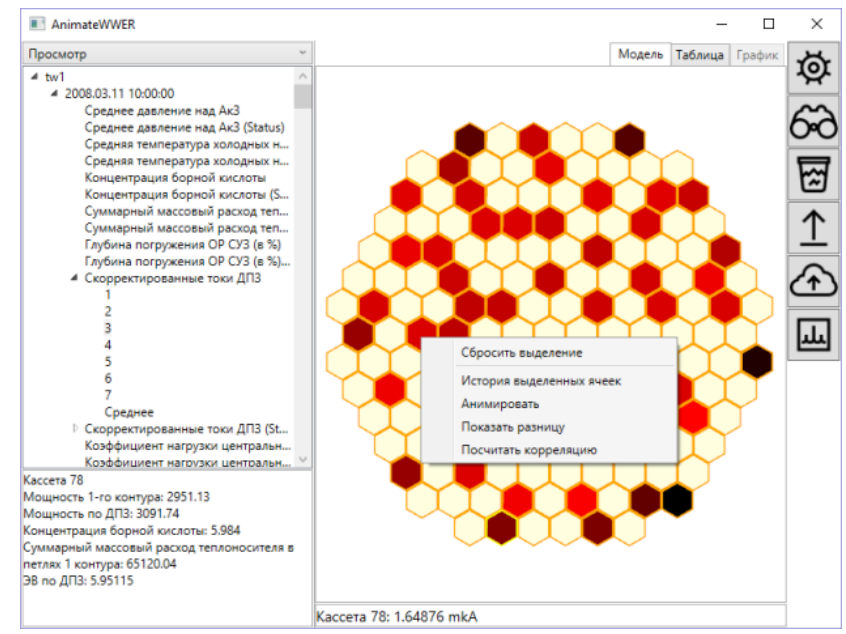

Figure 2: 2D renderer window after selecting the required information 
There was developed a $3 \mathrm{D}$ data flow animation module (fig. 3) that allows viewing changes in parameter values throughout the active zone in the form of animation with customizable parameters, a difference module for displaying the difference in parameter values for two different time slices, a module for displaying the history of cell values and a module for calculating the correlation of a parameter value [11].
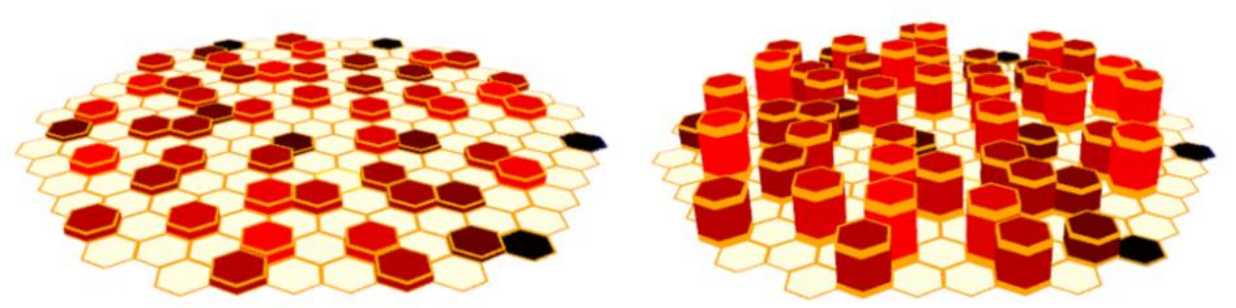

Figure 3: 3D visualization of the reactor core

From the point of view of express analysis of the archive, software [10] has been previously developed and presented which allows to export, preprocess, and visualize multidimensional data of the RBMK archive. This software was developed using effective modern data visualization tools. Since the archive is mostly multidimensional data, the problem of reducing the dimension of the space of variables was solved to present $2 \mathrm{D}$ or $3 \mathrm{D}$ visualization for further processing and making judgments about the safety of a nuclear reactor.

The first method used to solve the problem is the method of principal components. The multidimensional vector of parameters and maximum allowable values were projected onto the first three principal components, which, as a rule, reflected most of the variance. Further, the trajectory of the motion of the parameters in the main components was deduced. At the same time, the boundary of the maximum permissible values was visualized in the space of variables. Going beyond the settings of at least one of the parameters leads to an unplanned decrease in power, which can lead to an emergency.

In Fig. 4, you can see when parameters are approaching or crossing limits and analyze time slices to identify the cause. The color of the point for the time slice indicates the deviation of the values from the mathematical expectation in accordance with the scale. In the role of the missing lower (upper) limits, the minimum values over the entire (specified) time interval were chosen.

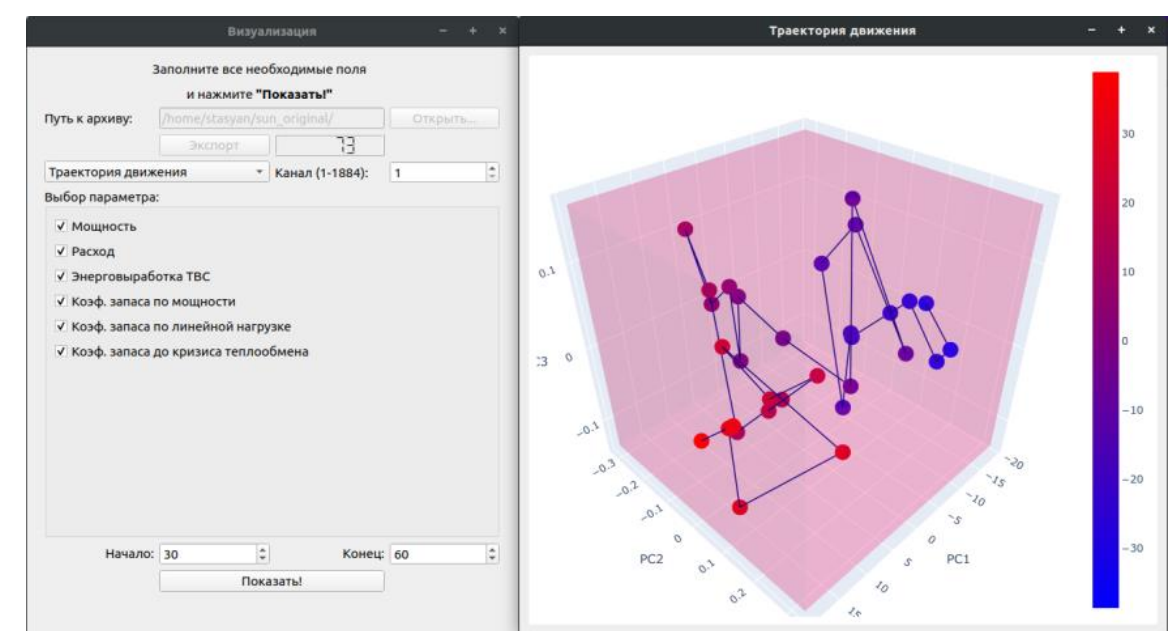

Figure 4: An example of using an application for visualizing the trajectory of movement

The second, no less popular method for visualizing multidimensional data, was the Chernoff Faces method. This approach allows to visualize multidimensional data using a 
human face for these purposes [15]. Individual parts of the face, such as eyes, eyebrows, nose, lips, depict the meanings of various signs, changing their size, shape or position. Usually, the Chernoff faces method is used when it is necessary to group (cluster) objects according to several characteristics, or when it is necessary to analyze presumably complex relationships between variables.

The result of visualization by the Chernoff faces method is observed in Fig. 5. Analyzing the results obtained, it is possible to draw conclusions when some of the parameters deviate greatly from their average values, when maximums or minimums are reached, or to find implicit relationships between the parameters (when visualizing more parameters). Such an approach, with the simultaneous use of a larger number of parameters, would make it possible to visually highlight clusters of similar facial expressions or find hidden dependencies of parameters among themselves.

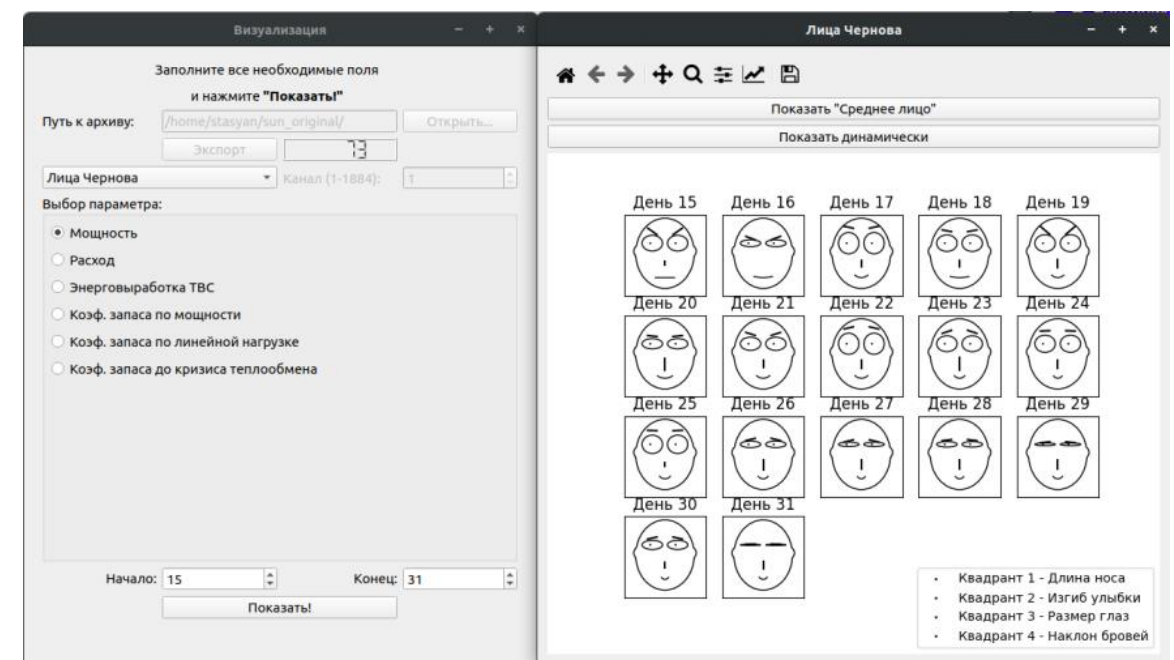

Figure 5: An example of using the application for visualization by the Chernoff faces method

Performing a visual analysis of the visualization result, you can manually select clusters of similar values, or outliers. For example, in Fig. 5, you can note that on days 17-25 the eyes on the face are greatly expanded, however, starting from day 26 , the eyes are narrow, which suggests that it is necessary to pay attention to this transition and analyze the archive in more detail on days 25-26. You can also pay attention to day 16 - you can notice that the nose on the face is practically absent, which indicates that there was some deviation on this day and a deeper analysis is needed to identify the causes. Similar outliers can be observed on days 15 and 19 - the eyebrows on the face are heavily frowned, which also indicates any deviations from the normal operation of the reactor.

Thus, this method allows you to visually identify deviations on the face. This is due to the peculiarity of human perception of the face and the ability to instantly detect even the slightest changes in it.

\section{Specification of the VVER reactor archive and data preprocessing}

Unlike the archives of the SKALA-MICRO system, the archives of the VVER reactor parameters are transmitted as a set of files with the .txt extension, distributed over directories. 


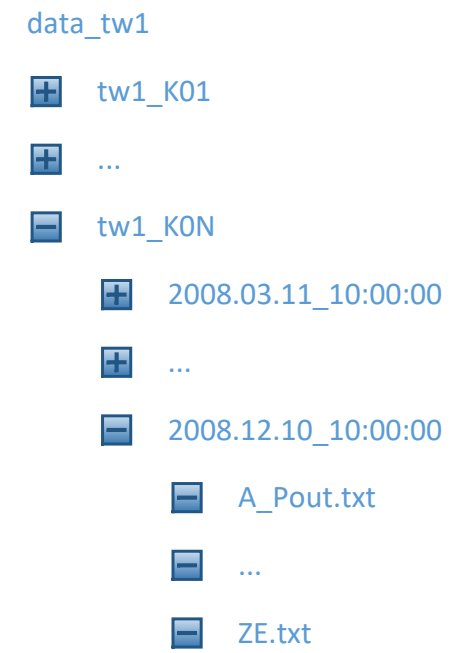

Figure 6: The structure of the VVER reactor archive

The name of the campaign is written in the name of the archive file, the root of the archive contains intermediate directories within one campaign, each of which contains directories whose names indicate a time slice. Each time slice contains a set of files with a .txt extension for each parameter. The archive structure is shown in Fig. 6.

Each file contains parameter values for that slice for each cassette. Bindings to cassettes are divided into three types: binding to the cassette number, binding to the KNI number, and binding to the control system number. Along with the archive of parameters, a text configuration file is supplied that defines the relationship between cassette numbers, KNI numbers and CPS numbers. Several lines from this file are presented in Table 1.

Table 1. Fragment from the configuration file of accordance between KNI and SUZ to cassettes

\begin{tabular}{|l|l|l|l|}
\hline Relation type & Value type & Cassette number & Related element number \\
\hline Kni_Pos & Int & 1 & 158 \\
\hline Kni_Pos & Int & 2 & 163 \\
\hline Kni_Pos & Int & 3 & 133 \\
\hline Kni_Pos & Int & 4 & 93 \\
\hline Kni_Pos & Int & 5 & 97 \\
\hline$\ldots$ & & & \\
\hline Suz_Pos & Int & 1 & 17 \\
\hline Suz_Pos & Int & 2 & 74 \\
\hline Suz_Pos & Int & 3 & 151 \\
\hline Suz_Pos & Int & 4 & 13 \\
\hline Suz_Pos & Int & 4 & 90 \\
\hline
\end{tabular}

Each file in the archive begins with a header containing the campaign name, cut time, measurement units and parameter name. Further along the lines, the values of the parameter 
for this slice are recorded with reference to the cassette number, KNI number or CPS number and possible reference to the level for parameters whose values can be multilevel. Determination of the type of binding is carried out analytically and depends on the number of records in the file. The configuration file compares the number of records in the file and the number of cassettes, KNI and CPS. The matched element number is a parameter bindable number.

Also, a file with the .pdf extension is attached to the archive, containing descriptions of each parameter that may be present in the archive.

To start working with the database, you need to execute a script to initialize the tables that store information about the unloaded archive. You also need to create a configuration file first.

The configuration files provided with the archive are recorded in the "config", "cassettes" and "parameters" tables. After successful configuration, the configured field in the "config" table is set to true.

For each parameter presented in the archive, a separate table is created, called "p1", "p2", etc., where the digit is the parameter id. Some parameters may not be present in the archive, therefore tables are created only for the present parameters. After creating a table of values for a certain parameter, the presence field in the "parameters" table for this parameter is set to true.

When reading the archive, it is sequentially written to the "campaigns" tables, "timepoints" and the table for a specific parameter.

The campaign parameters table is filled in as the archive is read. This table identifies the presence of parameters in a specific campaign. The table is primarily used to speed up the retrieval of data for display in the user interface.

Fig. 7 shows the structural model of the database of the archived values of the VVER reactor parameters.

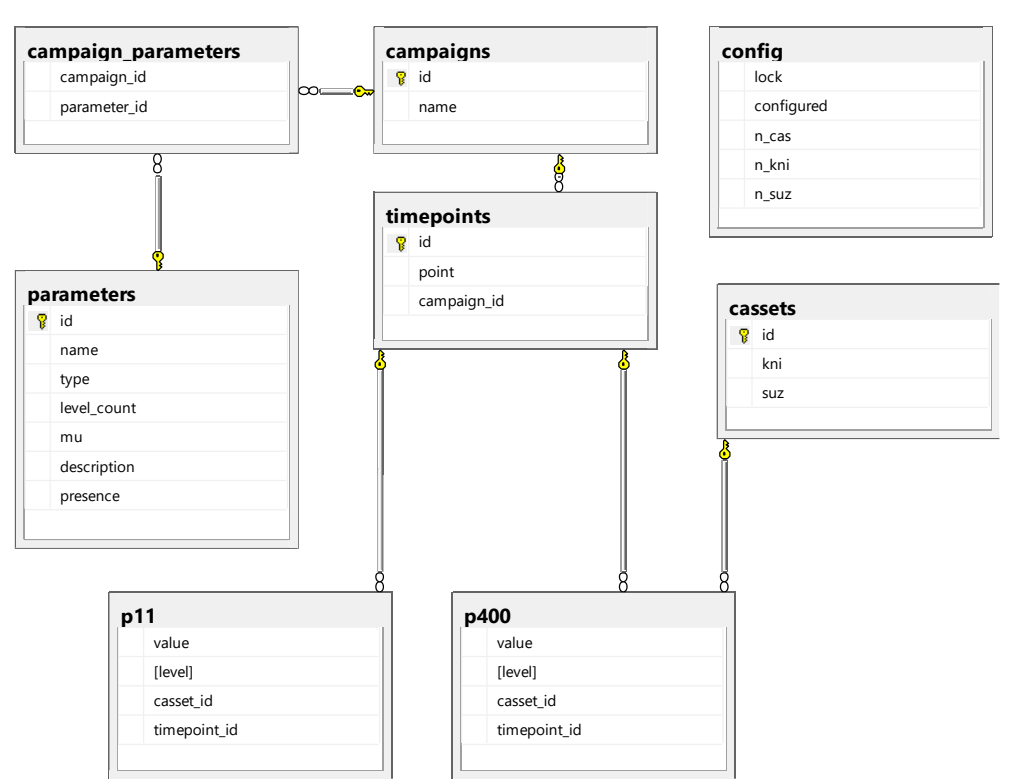

Figure 7: The structure of the database

Scripts were developed for reading parameters and further processing in order to build visual graphs. Also, a universal script was implemented for building the same type of charts.

The connection to the database from the program occurs through SQL queries, namely the pyodbc library. The third party pyodbc module makes it easy to access databases through the Open Database Connectivity (ODBC) programming interface.

Further, to retrieve the parameter from the database, you need to execute the SQL query using the above class using the pandas library. Pandas is a high-level Python library for data 
analysis. In the Python ecosystem, pandas is the most advanced and fastest growing library for data processing and analysis.

Thus, it is possible to extract parameters into convenient structures provided by the same pandas library - DataFrame. The structure is an indexed multidimensional array of values (Fig. 8).

\begin{tabular}{|c|c|c|c|c|c|c|c|}
\hline & point & value & level & casset_id & timepoint_id & id & campaign_id \\
\hline 0 & 2008-03-11 10:00:00 & 5.437390 & 4 & 2 & 152 & 152 & 1 \\
\hline 1 & 2008-03-12 10:00:00 & 3.028619 & 4 & 2 & 287 & 287 & 1 \\
\hline 2 & $2008-03-1310: 00: 00$ & 4.320083 & 4 & 2 & 85 & 85 & 1 \\
\hline 3 & $2008-03-14$ 10:00:00 & 4.601747 & 4 & 2 & 139 & 139 & 1 \\
\hline 4 & $2008-03-15$ 10:00:00 & 5.236040 & 4 & 2 & 159 & 159 & 1 \\
\hline$\ldots$ & $\ldots$ & $\ldots$ & $\ldots$ & ... & $\ldots$ & $\ldots$ & $\ldots$ \\
\hline 2177 & 2015-01-19 10:00:00 & 2.724344 & 4 & 2 & 1883 & 1883 & 1 \\
\hline 2178 & $2015-01-2010: 00: 00$ & 2.478261 & 4 & 2 & 2149 & 2149 & 1 \\
\hline 2179 & $2015-02-2110: 00: 00$ & 0.882122 & 4 & 2 & 2182 & 2182 & 1 \\
\hline 2180 & $2015-02-22$ 10:00:00 & 1.926554 & 4 & 2 & 2181 & 2181 & 1 \\
\hline 2181 & $2015-02-2310: 00: 00$ & 2.511921 & 4 & 2 & 2180 & 2180 & 1 \\
\hline
\end{tabular}

2182 rows $\times 7$ columns

Figure 8: A two-dimensional indexed DataFrame

To build graphs, a method was developed using the matplotlib and seaborn libraries. Matplotlib is a 2D (and 3D) graphics library for the python programming language that can be used to create high-quality drawings in various formats. Matplotlib is a python package module. Seaborn is a high-level API based on the matplotlib library. Seaborn contains more adequate default charting settings.

\section{Software package for express analysis of VVER reactor archive}

Based on the existing developments for carrying out express analysis in the field of visualization of the RBMK archive, software for the VVER archive was developed. The developed software makes it possible to analyze the archive by constructing high-altitude or time graphs and Chernoff faces, allowing to select the parameters and settings of the desired visualization. Fig. 9 shows the main window of the application. This complex allows connecting to a database storing pre-downloaded data and customizing the configuration of the desired visualization.

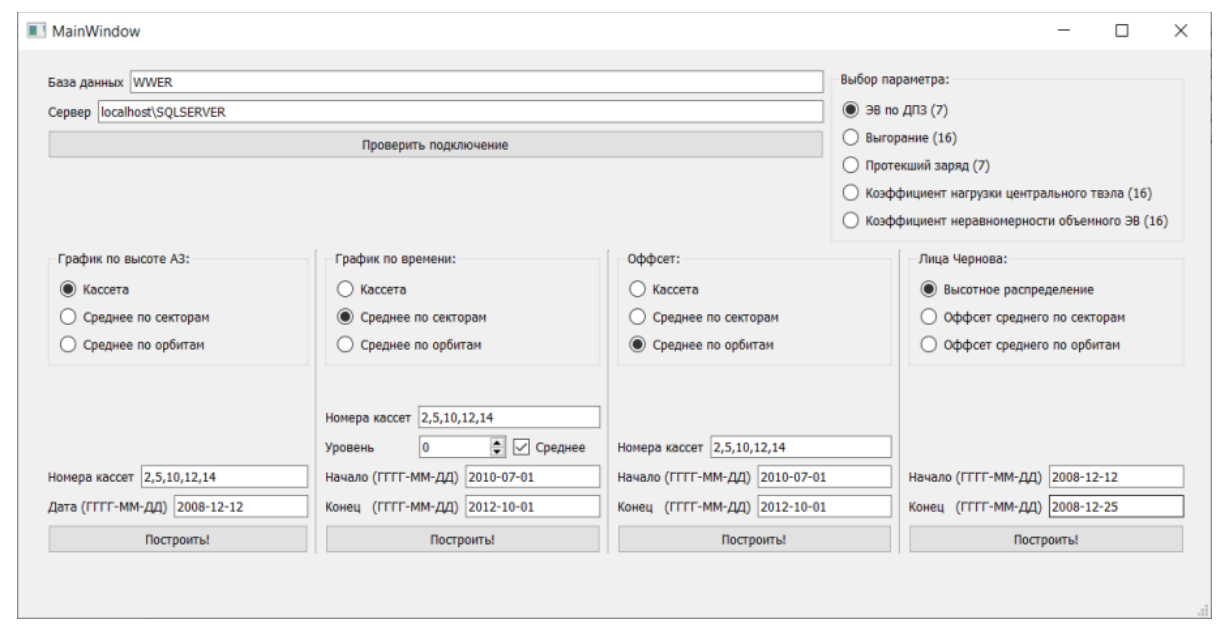

Figure 9: Main window of the implemented software 
To build any type of graphs offered by the complex, it is first needed to select the parameter to be visualized. At the moment, the complex supports the following parameters:

- Power generation by the DPZ;

- Burnout;

- Leaked charge;

- Load factor of the central TVEL.

At the same time, the program was developed considering the possibility of adding parameters for visualization.

Also, to reduce the number of displayed parameters and further visualization, the core was divided into 60-degree sectors of symmetry (Fig. 10).

For the same reasons, the active zone was divided into orbits separately in a similar way (Fig. 11). Further, for each sector (orbit), the parameter value is averaged for all TCs contained in the sector (orbit), and the average value is visualized in the form of graphs to study the behavior of generalized parameters over time.

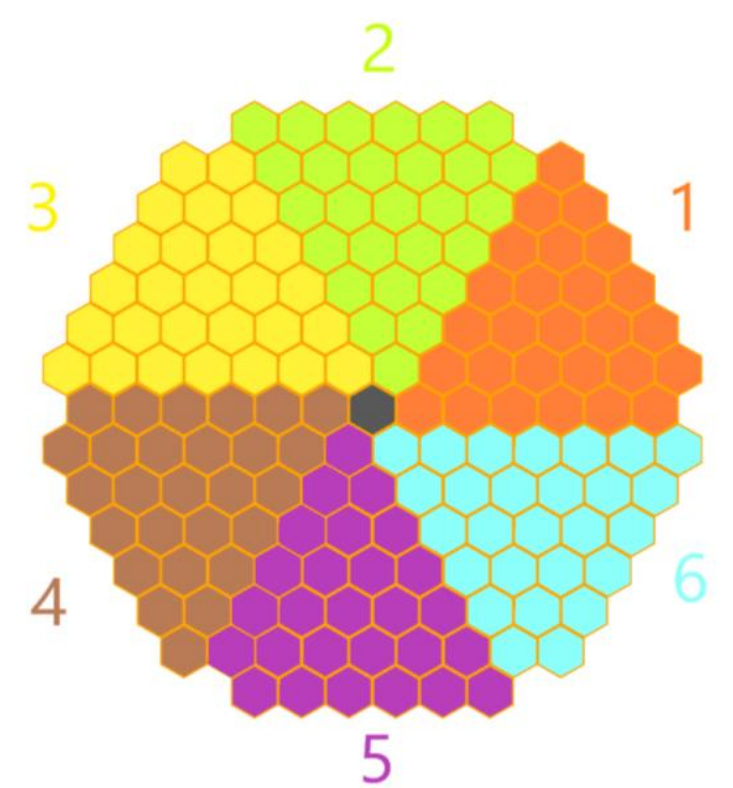

Figure 10: Division of the core into 6o-degree sectors of symmetry

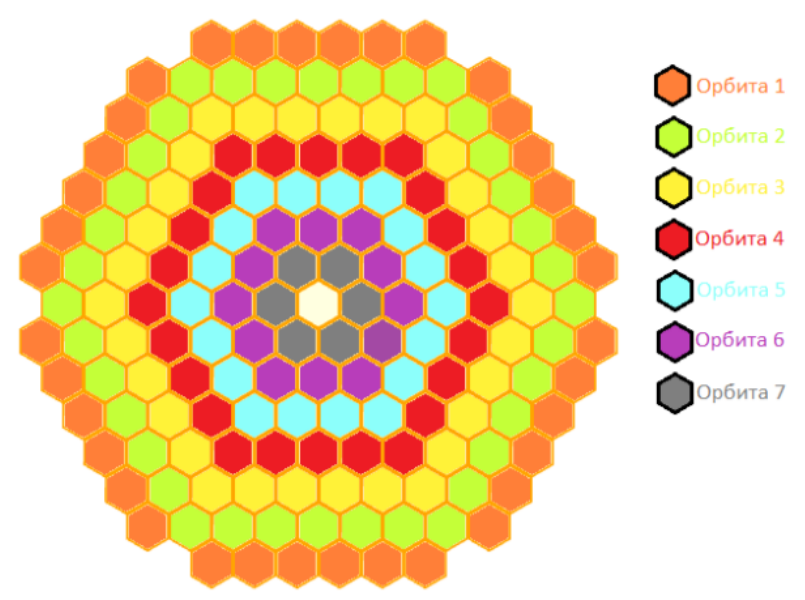

Figure 11: Division of the core into orbits

The developed software package can be conceptually divided into 4 parts. Each part is a separate type of visualization that can be built by preconfiguring the required configurations. 
Also, the developed software allows to work with graphics: change the scale, adjust colors and line types, save the graph as a graphic image.

At the same time, each of the visualizations allows to preprocess data for a more general visualization. The following options are available for preparing data for further visualization using one of the proposed methods:

- Cassette (output of the parameter value for the cassette);

- Sector average (sector-averaged parameter value);

- Orbital average (orbital-averaged parameter value);

- Height distribution (only for Chernoff faces);

- Offset of the average by sector (only for Chernoff faces);

- Orbital average offset (only for Chernoff faces).

Applying these methods, it is possible to draw conclusions when some of the parameters deviate greatly from their average values, when maximums or minimums are reached, or to find implicit relationships between the parameters (when visualizing more parameters).

1. Diagram of the parameter distribution over the height of the reactor core (Fig. 12). To build a graph, you need to configure the following parameters:

- Cassette numbers;

- Date in the format "YYYY-MM-DD".

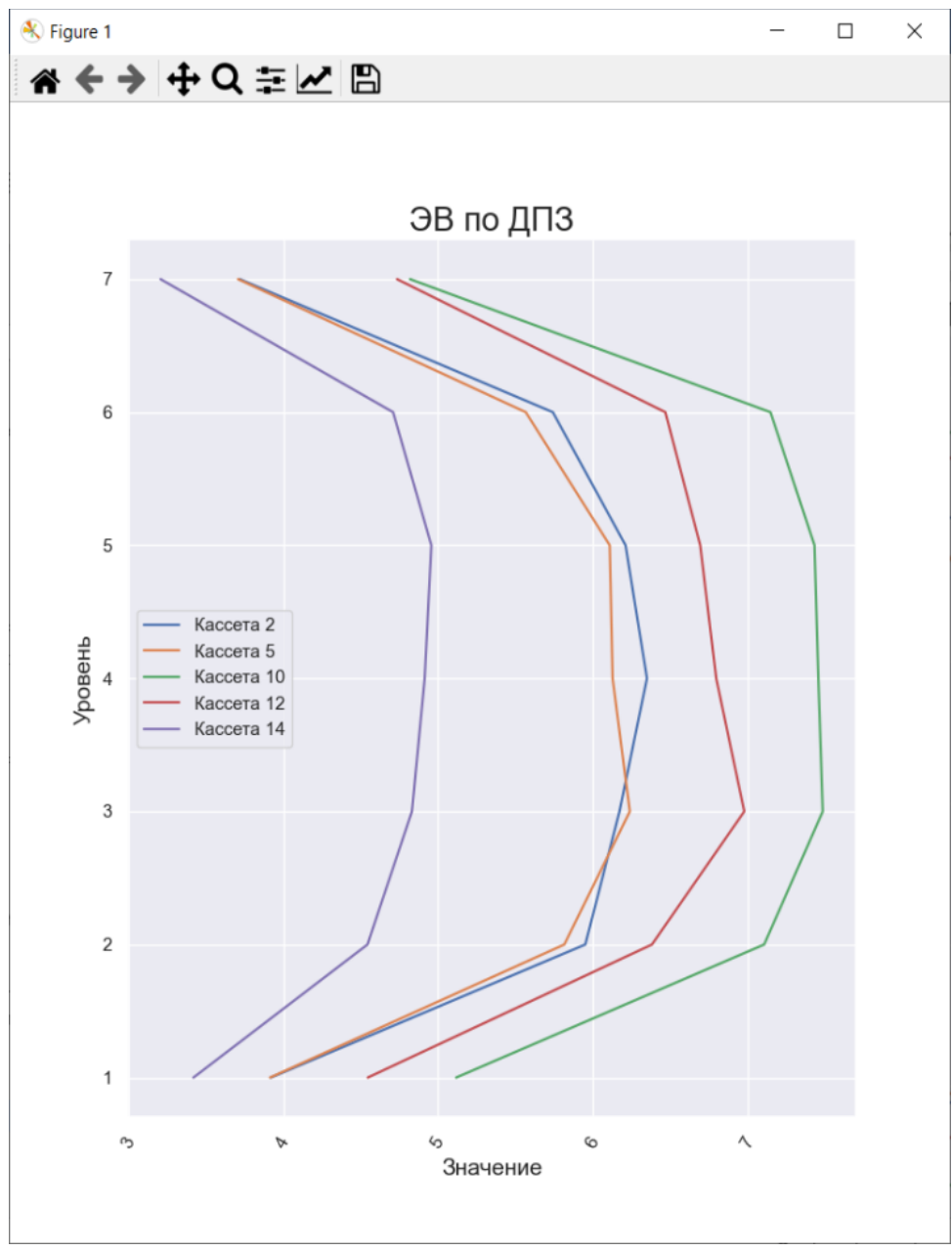

Figure 12: Diagram of the parameter distribution over the height of the reactor core

2. The timeline graph of the parameter (Fig. 13). To build a graph, you need to configure the following parameters:

- Cassette numbers;

- Level; 
- Start date of the timeline in the "YYYY-MM-DD" format;

- Date of the end of the timeline in the format "YYYY-MM-DD".

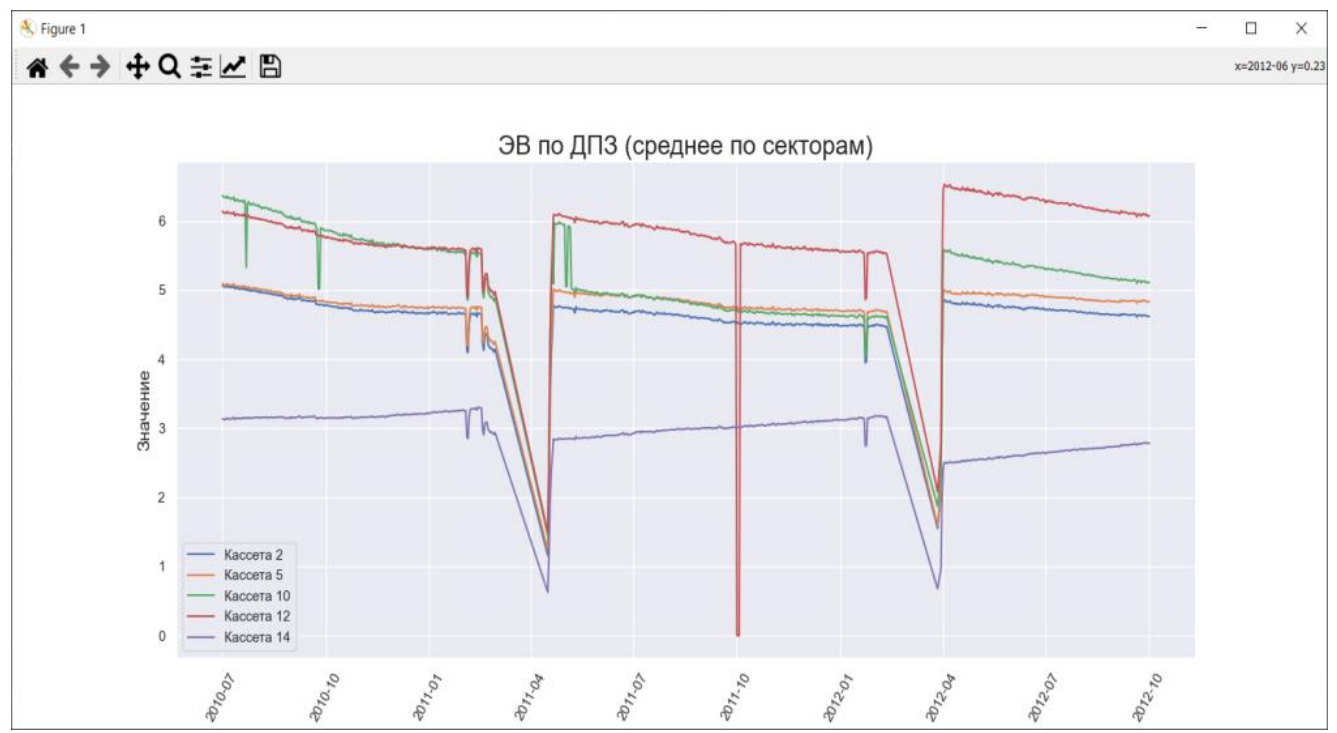

Figure 13: Parameter timeline

3. The timeline graph of parameter offset (fig. 14). To build a graph, you need to configure the following parameters:

- Cassette numbers;

- Start date of the timeline in the "YYYY-MM-DD" format;

- Date of the end of the timeline in the format "YYYY-MM-DD".

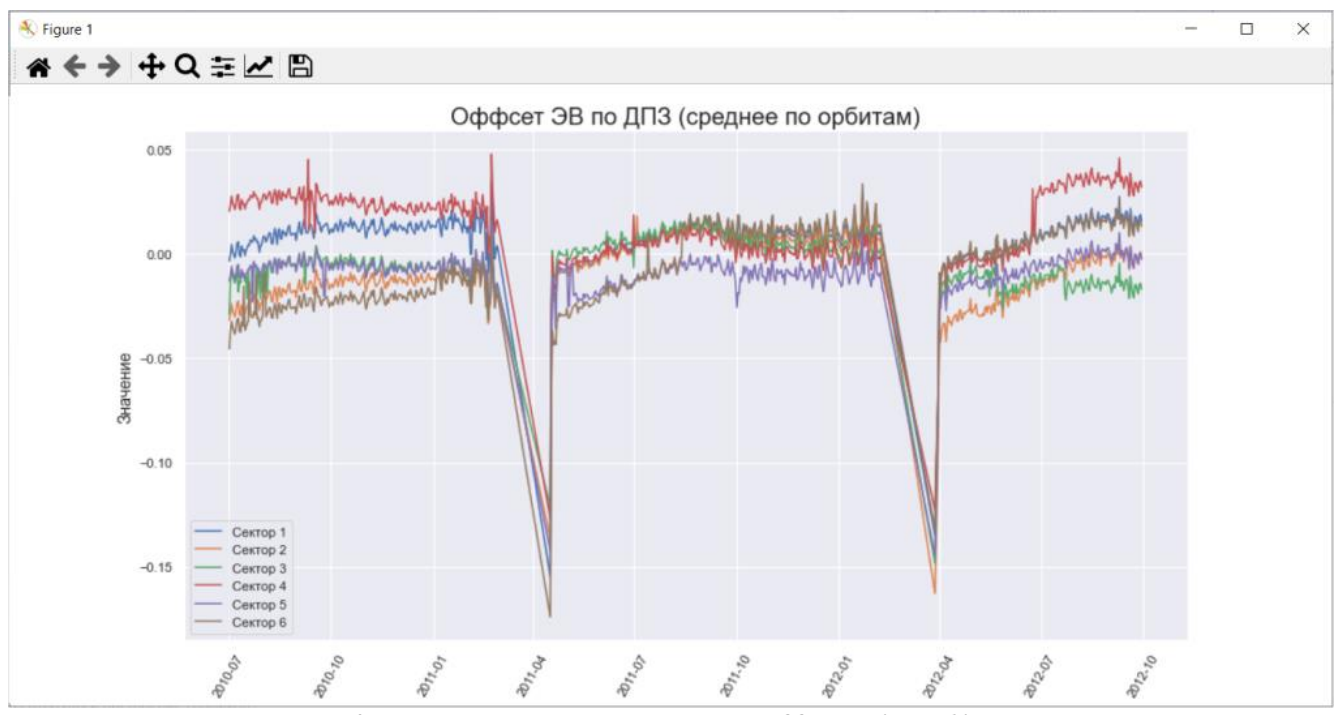

Figure 14: Parameter offset timeline

4. Visualization by Chernoff Faces method (Fig. 15). To build a graph, you need to configure the following parameters:

- Start date of the timeline in the "YYYY-MM-DD" format;

- Date of the end of the timeline in the format "YYYY-MM-DD". 


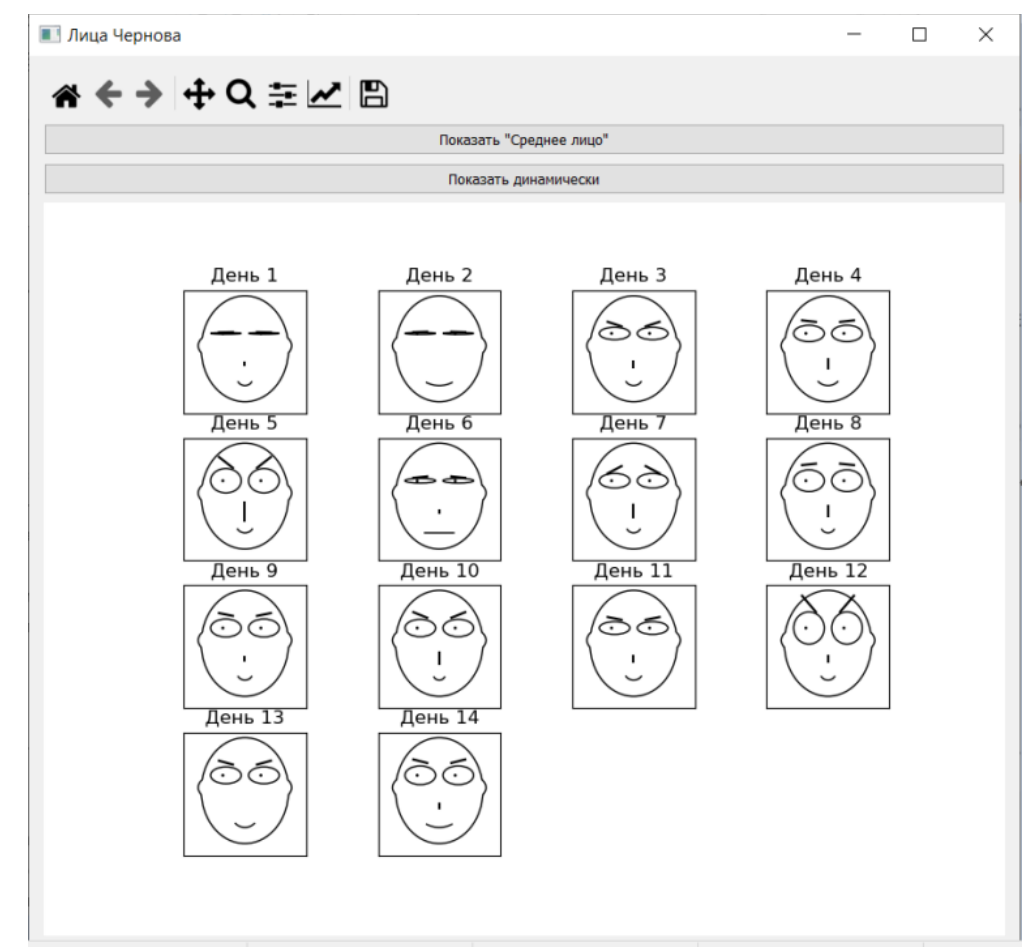

Figure 15: Static rendering with Chernoff faces

It should be noted that the type of visualization with the use of Chernoff faces also supports dynamic changes in the characteristics of the face, which allows visually, "in real time", to trace the dynamics of changes in the face in comparison with the "average face" face, that characteristics take average values for the selected time slice. An example of such visualization is shown in Fig. 16.

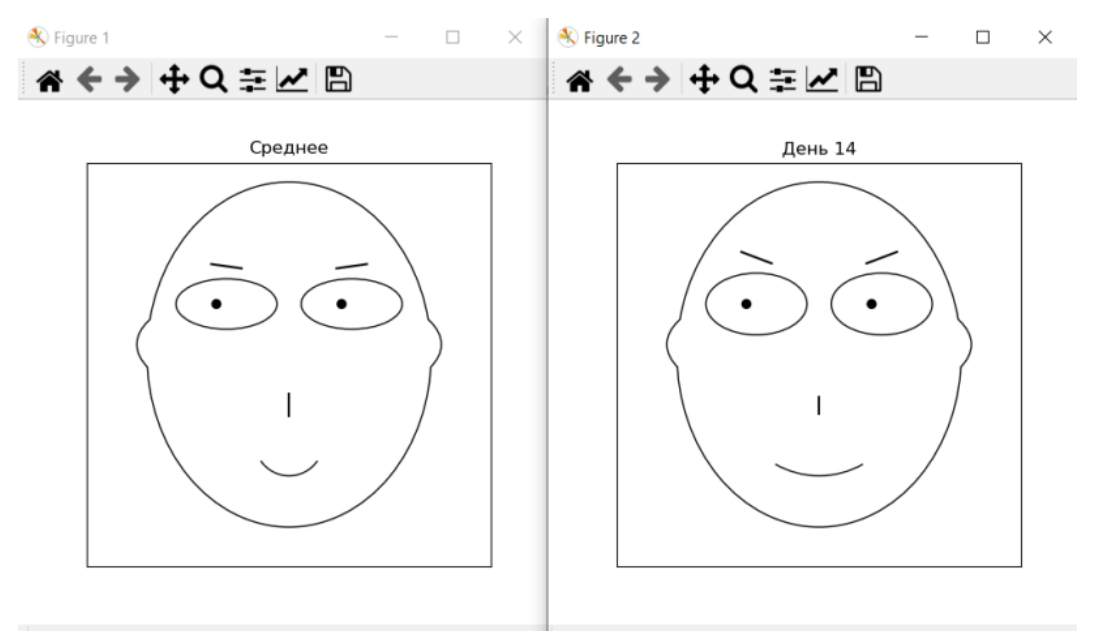

Figure 16: Dynamic rendering with Chernoff faces

\section{Conclusion}

This article presents a software package for visualizing the archive of a VVER-type nuclear reactor. This complex has an advantage over the previously developed ones in terms of performing express analysis and building dynamic visualization. With the help of this complex and the developed methods, it is possible to construct visualizations with the desired settings and, thus, to reveal anomalies or patterns in the behavior of parameters over time. Also, adapted visualization based on the Chernoff faces method allows to cluster generalized parameters, or to identify strong deviations, thereby speeding up the process of the analysis itself. After conducting a generalized study, it is possible to move on to a more detailed, in- 
depth study of the movement of individual parameters to identify the reasons for this behavior.

The novelty of this software is using of new methods applied to the archives of operational parameters of nuclear reactors. In this area, the use of new methods can reveal new patterns, as well as improve speed and quality of the analysis of the reactor behavior in different time slices (for example, during the day or throughout the year). Also, a structured approach based on the scientific visualization method allows the implementation of software that can be used both for post-analysis of the archive and for use by operational personnel. In this case, the software is developed in such a way that, when using various archives and configuration settings, a result can be obtained that can be interpreted for the initial object of consideration - the reactor.

Also, the implementation of new software systems for visualizing the archives of a nuclear reactor makes it possible to approach the solution of the analyzed problem from the point of view of an express analysis of the archive and thus conduct a study of multidimensional archive data using appropriate methods.

The proposed software can be used both by the operating personnel of the NPP as an auxiliary one in order to increase the efficiency of monitoring the operation of the power unit, and in order to analyze the existing archive database.

It is planned to continue working on improving the quality and stability of the developed software package, as well as expanding the functionality and adding new features.

\section{References}

[1] G. Tikhomirov, I. Saldikov, E. Malikova, L. Kuchenkova, V. Pilyugin. NRNU MEPhI experience in development and application of visualization software in nuclear power plants education. Scientific Visualization, 2012, volume 4, number 2, pp. 57-63.

[2] A.O. Bukalin, A.M. Zagrebaev, V.N. Samanchuk. Visualization of the process of neutron-physical calculation of a nuclear reactor. Scientific Visualization, 2020, volume 12, number 1, pp. 112-119, doi: 10.26583/sv.12.1.10

[3] I. Ivanov, S. Bychkkov, V. Druzhinin, I. Moiseyev, U. Shmonin, N. Shchukin. Application of visualization tools for analysis of statistical uncertainties in monte carlo calculations of channel-wise functionals in rbmk-1000. Scientific Visualization, 2012, volume 4, number 1, pp. 22-30.

[4] D. Zinakov. ReQt - software visualization and analysis of calculation results of RBMK-1000 reactor cores. Scientific Visualization, 2012, volume 4, number 1, pp. 31-41.

[5] Dollezhal' N.A., Emel'yanov I.Ya. Kanal'nyi yadernyi ehnergeticheskii reaktor. [Channel Nuclear Power Reactor] Atomizdat Publ., 1980.

[6] M.A. Abramov, V.I. Avdeev, E.O. Adamov et al.Edited by Yu.M. Cherkashova. Kanal'nyi yadernyi ehnergeticheskii reaktor RBMK.[Channel Nuclear Power Reactor RBMK] GUP NIKIEhT Publ., 2006. 632 p.

[7] Ovchinnikov F.Ya., Golubev L.I., Dobrynin V.D., Klochkov V.I., Semenov V.V., Tsybenko V.M. Ehkspluatatsionnye rezhimy vodo-vodyanykh ehnergeticheskikh yadernykh reaktorov. [Operational modes of water-cooled nuclear power reactors] Atomizdat Publ., 1975 .

[8] Nuclear Power Engineering. Problems. Solution. Edited by M.N. Strikhanov. Part 1. M.: Social Forecasts and Marketing Center, 2011. 424 p.

[9] A.M. Zagrebayev, R.N. Ramazanov. Nuclear Reactor RBMK Archive Data Visualization. Scientific Visualization, 2015, volume 7, number 2, pp. 1-11

[10] Compression and Visualization of the Operational Parameters Archive. Andrey Zagrebayev, Victor Pilyugin, Stanislav Ten. GraphiCon 2020. Computer Graphics and Machine Vision. pp. 1-11. doi: 10.51130/graphicon-2020-2-3-11 
[11] A.M. Zagrebayev, I.Yu. Leveev, V.V. Pilyugin, S. Ten. Creation and visualization of archives of operational RBMKs parameters and VVER reactors. Scientific Visualization, 2020. volume 12, number 4, pp. 33-45. doi: 10.26583/sv.12.4.04

[12] Yurova L.N., Naumov V.I., Savander V.I., Zagrebayev A.M. Kompaktnoe predstavlenie vynutrireaktornoi informatsii o potoke neitronov / Fizika yadernykh reaktorov. [Compact representation of in-reactor neutron flux information. / Physics of nuclear reactors.] Atomizdat Publ., 1975, number 4, pp. 19-23

[13] Alimov A.L., Shchadilov A.E. Optimal'noe adaptivnoe szhatie tsifrovykh soobshchenii po algoritmu kusochno-lineinoi approksimatsii. [Optimal adaptive compression of digital messages using piecewise linear approximation algorithm] Avtometriya Publ., 1983, number 3, pp. 14-18.

[14] A.M. Zagrebayev, V.A. Nasonova, N.V. Ovsyannikova. Matematicheskoe modelirovanie yadernogo reaktora pri sluchainykh vozmushcheniyakh tekhnologicheskikh parametrov. [Mathematical modeling of a nuclear reactor with random perturbations of technological parameters] NRNU MEPhI Publ., 2009. 400 p.

[15] Chernoff, H. The Use of Faces to Represent Points in K-Dimensional Space Graphically. Journal of the American Statistical Association 68 (342). 1973. pp. 361-368. doi: 10.1080/01621459.1973.10482434 